\title{
Ghost Signals In Allison Emittance Scanners
}

\author{
Martin P. Stockli ${ }^{1,2}$, M. Leitner ${ }^{3}$, D. P. Moehs ${ }^{4}$, R. Keller ${ }^{3}$, and R. F. Welton ${ }^{1}$ \\ 1) SNS, Oak Ridge National Laboratory, P.O. Box 2008, Oak Ridge, TN 37831, USA \\ 2) Department of Physics, University of Tennessee, Knoxville, TN 37996, USA \\ 3) SNS, Lawrence Berkeley National Laboratory, 1 Cyclotron Rd., Berkeley, CA, 94720, USA \\ 4) Fermi National Accelerator Laboratory, P.O. Box 500, Batavia, IL 60510, USA
}

\begin{abstract}
For over 20 years, Allison scanners have been used to measure emittances of low-energy ion beams. We show that scanning large trajectory angles produces ghost signals caused by the sampled beamlet impacting on an electric deflection plate. The ghost signal strength is proportional to the amount of beam entering the scanner. Depending on the ions, and their velocity, the ghost signals can have the opposite or the same polarity as the main beam signals. The ghost signals cause significant errors in the emittance estimates because they appear at large trajectory angles. These ghost signals often go undetected because they partly overlap with the real signals, are mostly below the $1 \%$ level, and often hide in the noise. A simple deflection plate modification is shown to reduce the ghost signal strength by over $99 \%$.
\end{abstract}

\section{INTRODUCTION}

The emittance of a particle beam is defined as the six-dimensional distribution of all position coordinates along the three configuration space directions and their associated velocity coordinates. It is normally projected into two-dimensional planes, $\left\{\mathrm{x}-\mathrm{x}^{\prime}\right\},\left\{\mathrm{y}-\mathrm{y}^{\prime}\right\}$, and $\left\{z-z^{\prime}\right\}$, reducing the emittance into three subsets.

The transverse subsets are measured with a slit that selects a narrow band from the beam at equidistant position coordinates, either $\mathrm{x}$ or $\mathrm{y}$. Downstream, a second slit or a wire harp typically samples the evolved particle distribution to determine the distribution of the trajectory angles x' or y', respectively, with which the ions passed the upstream slit. Wire harps can measure the distribution in single shots but are subject to sagging, variation in the wire size, variations in the surface condition that affects the secondary electron emission coefficient, and variations in the gain and bias of the different amplifiers. A single secondary slit combined with some kind of scanning mechanism guarantees a uniform response, promising more reliable data for the trajectory angle distribution if the beam is stable during the time-consuming scan.

Most of the particle beam is intercepted on the first slit, where it generates a variety of scattered primary and secondary particles. When some of the charged particles reach the current collection device, they create ghost signals, i.e., signals that are not truly representative of the $\mathrm{x} / \mathrm{x}$ ' distribution being probed. A wellknown example is slit scattering that alters the trajec- tory angle and possibly the charge of primary particles and can produce secondary charged particles. Ghost signals are normally small, but their appearance at extreme coordinate values can significantly alter the measured emittances. Accordingly, it is important to minimize all ghost signals. This paper discusses ghost signals that are characteristic for electrical sweep scanners and demonstrates their mitigation in an Allison scanner.

\section{ALLISON EMITTANCE SCANNERS}

Over the last 20 years, Allison scanners [1] have been implemented in many laboratories [2] to measure the emittance of low-energy ion beams. Allison scanners feature a base that supports two sets of slits, which can be aligned within tight tolerances. Electric deflection plates are located in the space between the slits, as shown in Fig. 1. A shielded Faraday cup with secondary electron suppression allows for reliable measurements of the small beam currents [3] that pass

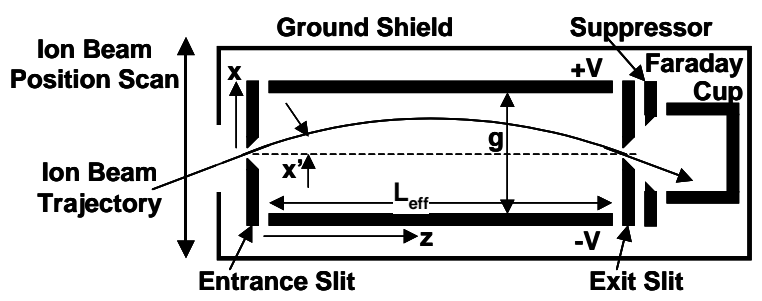

FIGURE 1. Schematic of an Allison emittance scanner. 
through both slits. A grounded shield that surrounds the entire assembly minimizes ghost signals from the beam stopped on the entrance slits. A stepper motor moves the entire assembly to scan the position distribution of the beam. At each position both deflection plates are charged with ramped voltages $\mathrm{V}$ of opposite polarity to determine the trajectory angle distribution of the beamlet passing through the entrance slit.

Figure 2 shows the emittance distribution of a broad, slightly converging $\mathrm{H}^{-}$beam from the SNS ion source [4] that was measured with our Allison scanner. The beam is measured as it emerges from an electrostatic lens [4], where aberrations cause tails at both ends of the position distribution, one of which is visible in Fig. 2.

The deflection voltage-to-angle conversion depends mainly on the deflection plate length $\mathrm{L}$ and the gap g between them. A fringing field correction [5] yields the more accurate effective length $\mathrm{L}_{\text {eff }}$, although the difference is normally small because $\mathrm{L}>\mathrm{g}$.

Integrating the transverse acceleration of the ions with charge $q$ and energy $q \cdot U$ yields the equation of motion $\mathrm{x}=\int \mathrm{v}_{\mathrm{x}} \cdot \mathrm{dt}=\mathrm{x}^{\prime} \cdot \mathrm{z}-\mathrm{V} \cdot \mathrm{z}^{2} /(2 \cdot \mathrm{g} \cdot \mathrm{U})$, where $\mathrm{x}$ is the transverse position coordinate, $\mathrm{z}$ is the axial distance from the entrance slit, and $x^{\prime}$ is the initial angle (in radians) of the ions passing through the entrance slit $(\mathrm{x}=0=\mathrm{z})$. To pass through the exit slit $\left(\mathrm{x}\left(\mathrm{z}=\mathrm{L}_{\mathrm{eff}}\right)=0\right)$, ions must enter with an angle $x^{\prime}=V \cdot L_{\text {eff }} /(2 \cdot g \cdot U)$, or, in other words, voltages of $\mathrm{V}=2 \cdot \mathrm{U} \cdot \mathrm{x}^{\prime} \cdot\left(\mathrm{g} / \mathrm{L}_{\mathrm{eff}}\right)$ are required to measure ions entering with an angle $x$ '.

The useful angular range is limited to $x^{\prime}{ }_{\text {max }}=2 \cdot g / \mathrm{L}$ because the transverse displacement cannot exceed $\mathrm{g} / 2$. This angle also determines the minimum taper that should be applied to the downstream side of both sets of slits to prevent scattering off the slit surface. Our Allison scanner features $\mathrm{L}=115 \mathrm{~mm}, \mathrm{~L}_{\text {eff }}=121 \mathrm{~mm}$, $\mathrm{g}=7 \mathrm{~mm}$, and hence $\mathrm{x}_{\text {max }}=0.115 \mathrm{rad}$.

\section{ALLISON SCANNER GHOST SIGNALS}

When the emittance scanner is aligned with the scanned beamlet, its center $\left(x^{\prime}(z=0)=0\right)$ impacts on the second slit as long as the applied voltage is less than $\mathrm{V}_{0}=\mathrm{U} \cdot(\mathrm{g} / \mathrm{L})^{2}$ or when sampling angles that are less than $x^{\prime}{ }_{0}=g /(2 \cdot L)=x^{\prime}{ }_{\max } / 4$. When these values are exceeded, the beamlet center impacts on a deflection plate at a distance $\mathrm{z}_{\mathrm{i}}=\left(\mathrm{g} \cdot \mathrm{L} /\left(2 \cdot \mathrm{x}^{\prime}\right)\right)^{1 / 2}=\mathrm{g} \cdot(\mathrm{U} / \mathrm{V})^{1 / 2}$. The angle of impact with respect to the deflection plate, $\alpha$, is given by $\tan (\alpha)=\left(2 \cdot x^{\prime} \cdot g / L\right)^{1 / 2}=(\mathrm{V} / \mathrm{U})^{1 / 2}$. These impact angles are small because $\mathrm{V}<<\mathrm{U}$.

The number of backscattered primary ions, as well as the number of secondary particles, increases rapidly with decreasing impact angle [6]. Practically all secondary particles have low kinetic energies. Therefore

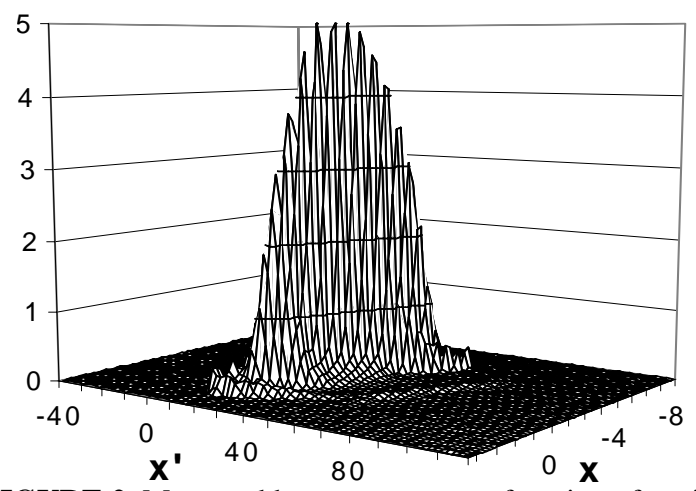

FIGURE 2. Measured beam current as a function of position $\mathrm{x}$ and trajectory angle $\mathrm{x}$ '.

charged secondary particles are quickly absorbed by the deflection plate with opposite polarity. Most scattered primary particles keep a large fraction of their forward momentum and therefore can reach the exit slit and generate ghost signals.

The emittance distribution of Fig. 2 is shown in the center of Fig. 3 as a density plot with gray tones that darken with increasing intensity. This central distribution is surrounded by an exclusively white zone, which indicates small signals that exceed the noise excursions. Further away, the white pixels are mixed with black pixels that indicate signals with a polarity opposite to the real signals. The zero of the scale was adjusted until the random-noise background in the lower left corner appeared as a random pattern with an equal mix of black and white. This method highlights ghost signals by revealing small deviations from randomnoise background.

Figure 3 shows a large black area of non-randomnoise background in the range between $\sim 30$ and $\sim 80$ mrad below the center of intense beamlets that pass through the entrance slit $(-5 \mathrm{~mm}<\mathrm{x}<2 \mathrm{~mm})$. A similar, but slightly smaller area is found above the center of the beamlets. The observed 30-mrad gap between the beamlet centers and the ghost signals matches the

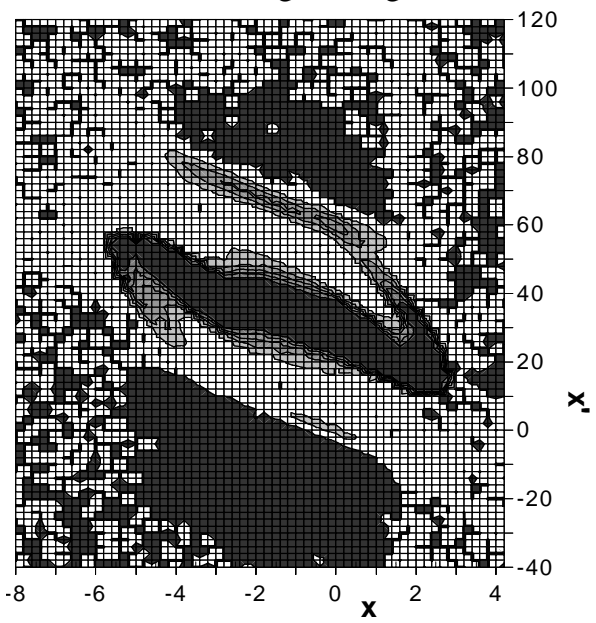

FIGURE 3. Density plot of the emittance data from Fig.2. 
predicted 29-mrad deflection of the beamlet centers before they hit the deflection plates. An accurate comparison would require this threshold to be convoluted with the distribution of the beam trajectory angles and the acceptance of the exit slit, suppressor, and Faraday cup system. When scanning for $\sim 30 \mathrm{mrad}$, the beamlet center impacts on the deflection plates a few millimeters in front of the exit slit with an impact angle of $\sim 3^{\circ}$. When scanning for $80 \mathrm{mrad}$, the beamlet center impacts on the deflection plates $\sim 50 \mathrm{~mm}$ in front of the exit slits with an impact angle of $\sim 6^{\circ}$. The increasing impact angle and the increasing distance from the exit slit cause the ghost signals to fade away. The asymmetry of the ghost signal ranges is caused by the $\sim 30$ mrad misalignment between the axes of the Allison scanner and the ion beam, as one can see in Fig. 3.

The ghost signals have an inverted polarity because of most $\mathrm{H}^{-}$ions being stripped during the backscattering process. Surface and near-surface scattering is often accompanied by a change of charge. Depending on the ions and their velocity, electron loss is likely for fast ions with low electron affinity. Electron gain is likely for highly charged ions. Accordingly, the net ghost signals have polarities that are equal or opposite to the real signals. Ghost signals that have the same polarity as the real signals are very difficult to detect unless emittance scanners are commissioned with highly collimated, narrow divergence beams.

\section{GHOST SIGNAL MITIGATION}

The small threshold of $\mathrm{x}^{\prime}{ }_{\max } / 4$ causes the ghost signals to overlap with the real signals as one can see in the asymmetry of the two tails. A separation would require the gap-to-length ratio $\mathrm{g} / \mathrm{L}$ to be increased. Maintaining the scanning range would require costly higher voltage supplies that are likely slower. Also likely, the deflection plate mount, the electrical connections, and vacuum feedthroughs require a redesign for significantly higher voltages. The gap has to remain smaller than the length $(\mathrm{g}<<\mathrm{L})$ to avoid the significant shortening of the effective length.

A dramatic improvement can be achieved by machining a staircase into the deflection plate surface. As

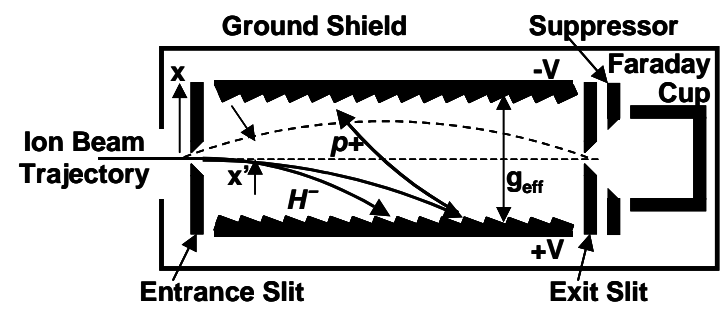

FIGURE 4. Staircase deflection plates bust ghost signals.

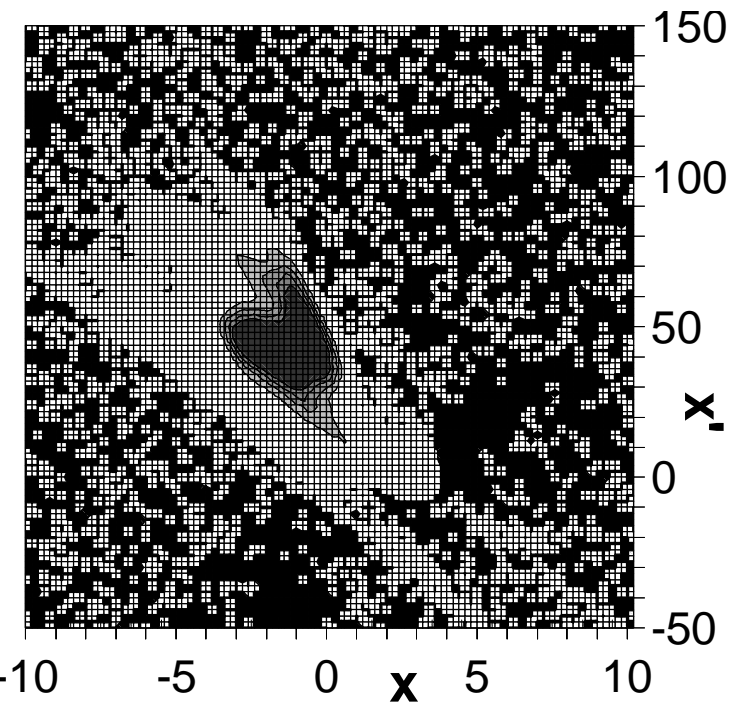

FIGURE 5. With stair-cased deflection plates, the emittance data exhibits random-noise background.

shown in Fig. 4, ions impact on the faces of the stairs with an almost normal impact angle. Backscattering is significantly reduced and results in trajectories that track backwards. The tilt angle must ensure that no ions impact on the flats of the stairs to avoid the increased backscatter probability at even shallower angles. The beamlet centers encounter the largest trajectory angle, $\alpha_{\max }$, when scanning for the maximum useful angle $x^{\prime}$, thus $\tan \left(\alpha_{\max }\right)=2 \cdot \mathrm{g} / \mathrm{L}$. Half of the beam's divergence and misalignment allowance need to be added. After adding another 50\% safety margin, we selected $70^{\circ}$ for the faces and $20^{\circ}$ for the flats of the stairs. After the modification, the emittance data show a central distribution that is surrounded by randomnoise background, as seen in Fig. 5.

The steps of our stairs are $1 \mathrm{~mm}$ high and $3 \mathrm{~mm}$ apart. After the steps were machined, a small, final cut was taken to obtain sharp edges that are $\sim 25 \mu \mathrm{m}$ wide. The edge width-to-separation ratio and the roughness of the edge surfaces suggest a ghost signal suppression in excess of 99\%. We approximate the new effective deflection plate gap geff with the sum of the gap between the ridges and height of one step.

\section{EMITTANCE ANALYSIS}

Adding a miniscule $0.01 \%$ bias to the ghost-free data increases the rms emittance by $33 \%$ when calculated from the raw data. More reliable rms-emittance estimates require an analysis with SCUBEEx, the SelfConsistent, UnBiased Elliptical Exclusion method [7], part of a free emittance analysis code [8]. It uses ellipses to separate the real signals from areas that contain pure background. In this work the Twiss parameters of 


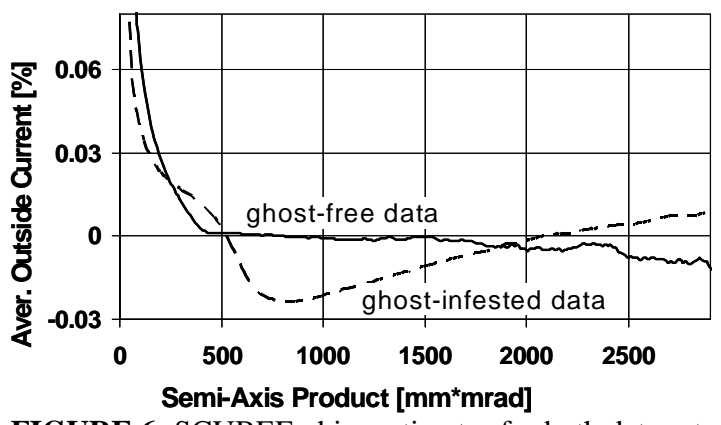

FIGURE 6: SCUBEEx bias estimates for both data sets

tightly fitting ellipses were determined from the data that exceeded a 10\% threshold. Figure 6 shows the average current measured outside such an ellipse as a function of its semi-axis product. When all real signals of the ghost-free data are included at $430 \mathrm{~mm} \cdot \mathrm{mrad}$, the average outside current no longer changes when increasing the ellipse size. However, statistical fluctuations become evident above $1000 \mathrm{~mm} \cdot \mathrm{mrad}$. Therefore the values in the intermediate range represent selfconsistent bias estimates. No such self-consistent bias estimates can be established for the ghost-infested data because the ghost signals significantly alter the average outside current.

Likewise, Fig. 7 shows the rms-emittance estimates as a function of the semi-axis product of the same ellipses, as in Fig. 6. These estimates are calculated from the data within the ellipse after subtracting the bias estimated from the average current found outside the ellipse. Again, when all real ghost-free signals are included above $430 \mathrm{~mm} \cdot \mathrm{mrad}$, the rms-emittance estimate no longer changes when increasing the ellipse size. And once more, statistical fluctuations become evident above $1000 \mathrm{~mm} \cdot \mathrm{mrad}$. Accordingly, the values in the intermediate range represent a self-consistent estimate of the semi-axis product of the normalized rms emittance, namely $0.115 \pm 0.002 \mathrm{~mm} \cdot \mathrm{mrad}$.

As before, SCUBEEx cannot self-consistently estimate the rms emittance of the ghost-infested data. Small ellipses underestimate the rms emittance because the ghost signals reduce the signals in the tails. Large ellipses underestimate the rms emittance because the negative contributions from the ghost signals

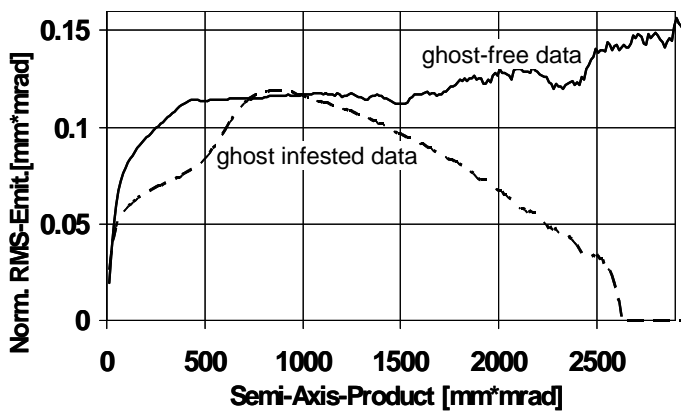

Figure 7. SCUBEEx rms-emittance estimates

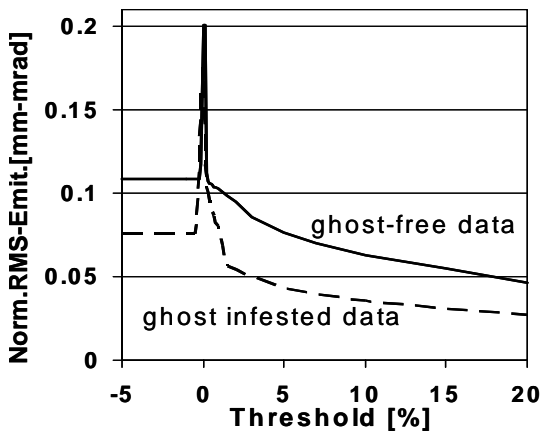

Figure 8. Rms-emittance estimates using thresholds

with large $x$ ' start to dominate until they exceed all positive contributions at $2620 \mathrm{~mm} \cdot \mathrm{mrad}$ where the rms emittance becomes imaginary. The two effects discussed previously could cause intermediate ellipses to underestimate the rms emittance, while an overestimation could be caused by the bias underestimation observed in Fig. 6. The two data sets have been measured under very different conditions. It is therefore purely coincidental that the rms-emittance peak value of the ghost-infested data matches the plateau value of the ghost-free data.

Figure 8 shows the rms emittance estimated from thresholded data. Applying a 1, 5, or $10 \%$ threshold underestimates the rms emittance of the ghost-free data by 11,39 , or $46 \%$, respectively. The ghost infested data reveal much stronger threshold dependence because the ghost signals significantly reduce the signals in the tails. The lack of a self-consistent rmsemittance estimate prohibits the evaluation of the associated errors, but its threshold dependence suggests errors that could be twice as high.

\section{REFERENCES}

1. P. W. Allison, J. D. Sherman, and D. B. Holtkamp, IEEE Trans. on Nucl. Sci. NS-30, 2204-2206 (1983).

2. e.g. M. Dombsky et al., Rev. Sci. Instrum. 60, 1170-1172 (1998); D. Wutte, M. A. Leitner, and C. M. Lyneis, Physica Scripta T92, 247-249 (2001); Y. J. Kim et al, Rev. Sci. Instrum. 75, 1681-1683 (2004).

3. e.g. M. P. Stockli and S. Winecki, Physica Scripta T71, 164-174 (1997).

4. R. Keller et al., Rev. Sci. Instrum. 73, 914-916 (2002).

5. H. Wollnik and H. Edwald, Nucl. Instr. and Meth 36, 93104 (1965) and its Ref. 2.

6. O. S. Oen and M. T. Robinson, Nucl. Instr. and Meth 132, 647-653 (1976).

7. M. P. Stockli, R. F. Welton, and R. Keller, Rev. Sci. Instrum. 75, 1646-1649 (2004); M. P. Stockli et al. in Production and Neutralization of Negative Ions and Beams, M. P. Stockli, edt., AIP Conference Proceedings 639, Melville, New York, 2002 pp. 135-159; R. F. Welton et al. in ibid, pp. 160-174.

8. https://www.sns.gov/APGroup/Codes/EAS/eas.htm. 